\title{
Padrão espacial de espécies madeireiras da Amazônia pelo método de coordenadas cartesianas e espaciais
}

\author{
Tiago Monteiro Condé ${ }^{1 *}$, Helio Tonini ${ }^{2}$, Fernando da Silva ${ }^{3}$, Paulo Eduardo Barni ${ }^{1}$, Carlos Henrique Souza Celes ${ }^{4}$ \\ Raquel Fernandes de Araujo ${ }^{4}$, Moacir Alberto Assis Campos ${ }^{4}$, Dirceu Lucio Carneiro de Miranda ${ }^{3}$
}

\author{
1Universidade Estadual de Roraima, Av. Senador Helio Campos, s/n, CEP 6937-3000, Rorainópolis, RR, Brasil \\ ${ }^{2}$ Embrapa Agrossilvipastoril, Rodovia MT 222, Km 2,5, CEP 78550-970, Sinop, MT, Brasil \\ ${ }^{3}$ Universidade Federal de Mato Grosso, Av. Alexandre Ferronato, 1200, CEP 78550-000, Sinop, MT, Brasil \\ ${ }^{4}$ Instituto Nacional de Pesquisas da Amazônia, Av. André Araújo, 2936, CEP 69060-001, Manaus, AM, Brasil
}

"Autor correspondente:
tiagonafloresta@gmail.com

Termos para indexação: Sistema de informação geográfica Manejo florestal

Índice do vizinho natural

\section{Index terms:}

Geographic information system

Forest management

Natural neighbor index

\section{Histórico do artigo:}

Recebido em 18/11/2015

Aprovado em 13/05/2015

Publicado em 30/06/2016

doi: $10.4336 / 2016 . p f b .36 .86 .1111$
Resumo - A utilização de sistemas de informação geográfica (SIG) no planejamento florestal possibilita a análise e o reconhecimento de padrões espaciais das espécies florestais em perfil bidimensional e tridimensional. O objetivo deste estudo foi demonstrar a eficiência do método de coordenadas cartesianas e espaciais (MCCE), método de correção da localização das coordenadas UTM das árvores em concordância com a localização de campo ou cartesianas $(\mathrm{X}, \mathrm{Y})$, aliado ao cálculo do índice do vizinho natural (ANND) no reconhecimento e análise de padrões espaciais de quatro espécies comerciais madeireiras em área de manejo florestal em Caracaraí, RR, Brasil. O ANND pressupõe completa aleatoriedade espacial.Simulações foram realizadas em 9 ha, subdivididos em 100 subparcelas de $100 \mathrm{~m}^{2}$ cada. Foram coletados: o diâmetro (DAP $\geq$ $10 \mathrm{~cm}$ ), alturas comercial e total, volume comercial e as coordenadas cartesianas (X,Y) e espaciais (UTM). Foram observados padrões espaciais aleatórios para Eschweilera bracteosa e Manilkara huberi. Os padrões espaciais dispersos e raros foram mais observados em Cedrelinga cateniformis e Dinizia excelsa. O MCCE demonstrou ser um método eficiente para o reconhecimento e análise de padrões espaciais de espécies nativas da floresta tropical amazônica, facilitando o planejamento florestal mediante simulações 2D e 3D da floresta.

\section{Spatial pattern of Amazonian timber species using cartesian and spatial coordinates method}

\begin{abstract}
Geographic information system (GIS) applied to forest analysis permit the recognition and analysis of spatial patterns of species in two and three dimensional. The aim of this study to demonstrate the efficiency of cartesian and spatial coordinates method (MCCE), method of correcting UTM coordinates of trees location in accordance with the location of field or Cartesian (X,Y), combined with natural neighbor index (ANND) in recognition and analysis of spatial distribution patterns of four commercial timber species in forest management in Caracaraí, Roraima State, Brazil. Simulations were performed on 9 ha, divided into 100 plots of $100 \mathrm{~m}^{2}$ each. Collected data were $\mathrm{DBH} \geq 10 \mathrm{~cm}$, commercial and total heights, cartesian coordinates $(\mathrm{X}, \mathrm{Y})$ and spatial coordinates (UTM). Random spatial patterns were observed in Eschweilera bracteosa and Manilkara huberi. The dispersed and rare spatial patterns were observed in Dinizia excelsa and Cedrelinga cateniformis. MCCE proved to be an efficient method in the recognition and analysis of spatial patterns of native species from Amazon rain forest, as forest planning becomes easier by $2 \mathrm{D}$ and $3 \mathrm{D}$ simulations.
\end{abstract}




\section{Introdução}

Um grande problema enfrentado em inventários florestais de floresta densa na Amazônia é a precisão na localização espacial georreferenciada por meio de GPS de árvores com diâmetros inferiores ao diâmetro mínimo de corte $(\mathrm{DMC} \geq 50 \mathrm{~cm})$. Árvores com diâmetro entre 5 a $30 \mathrm{~cm}$ representam a maioria dos indivíduos em florestas nativas (Souza et al., 2003; Condé \& Tonini, 2013) e geralmente são ignoradas em inventários florestais destinados à supressão vegetal ou "corte raso" na Amazônia, onde o diâmetro de exploração geralmente é maior do que $30 \mathrm{~cm}$ (Gimenez et al., 2015). Situações de agrupamento de árvores com diâmetro entre 5 a 30 $\mathrm{cm}$, como regiões de clareiras, indivíduos com vários fustes, espécies de crescimento em touceira, etc., podem apresentar falsos padrões espaciais quando associados aos erros de GPS em florestas naturais, ocasionando a perda de precisão no posicionamento de árvores e gasto de tempo no planejamento e execução de atividades relacionadas ao manejo florestal.

$\mathrm{Na}$ Amazônia, estimativas de diversidade florística e volumetria foram realizadas com base em grandes inventários florestais de cunho comercial, como os da FAO e do Projeto Radam Brasil, adotando geralmente o critério de inclusão de árvores com diâmetro superiores a $25 \mathrm{~cm}$ (Fearnside, 2000; ter Steege et al., 2006; Nogueira et al., 2008). Entretanto, inventários considerando todas as formas de vida em diferentes níveis de inclusão de diâmetro são minoria, como o da Reserva Ducke em Manaus (Ribeiro et al., 1999), os realizados pelo Serviço Florestal Brasileiro (Serviço Florestal Brasileiro, 2016), dentre outros. Quanto mais detalhada é a amostragem em um inventário florestal em nível de inclusão de espécies, maior será a demanda de precisão do posicionamento individual das amostras para representar fielmente os padrões espaciais interespecíficos.

O padrão espacial de uma espécie é representado por sua distribuição na área em estudo, em termos de frequência de ocorrência dentro das unidades amostrais (Jankauskis, 1990), ocorrendo de forma aleatória, uniforme ou dispersa (Krebs, 1999). Diversos índices clássicos de distribuição espacial, como MacGuinnes, Fracker e Brischle, Morisita e Payandeh (Rossi \& Higuchi, 1998; Nascimento et. al., 2001; Queiroz et al., 2007) e modelos espaciais que consideram as condições edafoclimáticas, competição, densidade, características hídricas e irradiação solar têm sido utilizados na compreensão de como as espécies florestais se distribuem em diversos ambientes (Turner \& Tjørve, 2005; ter Steege et al., 2013; Yang et al., 2013).

A modelagem espacial de populações de espécies florestais em ambientes de SIG possibilita uma visualização mais realística da natureza, auxiliando o entendimento das causas e processos ecológicos naturais, recriando ambientes em plataformas computacionais e facilitando simulações. Coordenadas geográficas e altimetria oriundos de GPS permitem o mapeamento da hidrografia, relevo, planejamento florestal e análise da espacialização de variáveis de interesse florestal (Figueiredo et al., 2007). Esta abordagem gera informações mais detalhadas e precisas sobre a localização das árvores, trazendo ganhos reais na determinação de padrões espaciais das espécies e suas relações ecológicas, o que auxilia sobremaneira o planejamento e execução de operações florestais.

A estrutura e composição florestal podem ser analisadas em perfil bidimensional (2D) e tridimensional (3D) (Figueiredo et al., 2007; Wezyk, 2012; Koch, 2012). O perfil 2D é realizado mediante a projeção vertical e horizontal das espécies e o perfil 3D representa a estrutura e composição das árvores em vertical, horizontal e profundidade (Koch, 2012). O último permite a observação de uma visão mais realista do ecossistema (Figueiredo et al., 2007; Koch, 2012; Wezyk, 2012). Esta abordagem em ambientes de SIG tem demonstrado que a inserção da análise espacial implica em questionamentos sobrea análise de padrões espaciais por métodos clássicos.

O objetivo deste estudo é demonstrar a eficiência do método das coordenadas cartesianas e espaciais (MCCE) aliado ao cálculo do índice do vizinho natural (ANND) no reconhecimento e análise de padrões espaciais de quatro espécies madeireiras em área de manejo florestal em Caracaraí, RR.

\section{Material e métodos}

O estudo foi realizado em ambiente de Floresta Ombrófila Densa de terra firme, em área sob manejo florestal da empresa Madeireira Vale Verde Ltda. no município de Caracaraí, RR. A topografia possui altitude variando de 60 a $120 \mathrm{~m}$. Os solos mais encontrados são: Argissolo Vermelho-Amarelo e Latossolo VermelhoAmarelo.O clima, segundo a classificação de Koeppen, é tropical chuvoso sem estação seca (Af), com precipitação pluviométrica média de $1.750 \mathrm{~mm}$ (Condé \& Tonini, 2013). 
Foram analisados os padrões espaciais de quatro espécies madeireiras: angelim-ferro (Dinizia excelsa (Ducke), Fabaceae); cedrorana (Cedrelinga cateniformis (Ducke) Ducke, Fabaceae); maçaranduba (Manilkara huberi (Ducke) A. Chev., Sapotaceae) e matá-matáamarelo (Eschweilera bracteosa (Poepp. ex O. Berg) Miers, Lecythidaceae), mediante o cálculo do índice do vizinho natural por meio da distância euclidiana média (average nearest neighbor distance - ANND), usando o programa ArcGIS 9.3. O ANND foi calculado utilizando as equações 1 a 3 (Environmental Systems Research Institute, 2008).

$$
\begin{aligned}
& \overline{D O}=\sum_{i=1}^{n} d_{i} / n \\
& \overline{D E}=0,5 / \sqrt{\frac{n}{A}} \\
& A N N=\overline{D O} / \overline{D E}
\end{aligned}
$$

Em que: $A N N=$ proporção ou taxa média do índice do vizinho natural em porcentagem; $\overline{D O}=$ distância média observada entre um ponto ou amostra e seu vizinho mais próximo;distância média esperada entre pontos ou amostras com padrão aleatório de seleção; $\overline{D E}=$ distância entre o ponto ou amostra $\mathrm{i}$ e outras amostras próximas; $d_{i}=$ número de pontos ou amostras do estudo; $A=$ área total do estudo.

A hipótese nula é a completa aleatoriedade espacial, ou seja, todas espécies analisadas apresentam padrão aleatório (Environmental Systems Research Institute, 2008). A rejeição ou aceitação da hipótese foi determinada com base nos valores de desvio padrão $(z)$ e probabilidade $(p)$ associada à distribuição normal com determinado nível de confiança $(90 \%, 95 \%$ e 99\%). Quando o valor de $\mathrm{p}$ é pequeno $(\mathrm{p}<0,01)$, onde há rejeição da hipótese de nulidade, temos duas alternativas, valores de $z$ são positivos, equivalendo a uma distribuição agregada, e valores de $z$ são negativos, representando uma distribuição dispersa, em ambos os casos indicando que provavelmente o padrão espacial observado não decorre de processos aleatórios, ou seja, possivelmente fatores ambientais não aleatórios como altitude, solo e topografia determinam os padrões observados.

Foram realizadas simulações com dados de 9 parcelas de 1 ha $(100 \mathrm{~m} \times 100 \mathrm{~m})$, subdivididas em 100 subparcelas de $10 \mathrm{~m}$ x $10 \mathrm{~m}$, instaladas conforme descrito em Silva et al. (2005). A área de estudo foi georreferenciada
(DATUM SAD69; Zona 20N; Coordenadas UTM: E 710.000 N 200.000). Foram coletados: diâmetro a $1,30 \mathrm{~m}$ do solo $(\mathrm{DAP}) \geq 10 \mathrm{~cm}$ com fita métrica, alturas comercial (AC = distância entre o solo e a inserção da copa, em m) e total (AT = distância entre o solo e o ápice da copa, em m) com a utilização do Hipsômetro (Vertex IV, Haglöf, Sweden), coordenadas cartesianas ou coordenadas de campo $(\mathrm{X}, \mathrm{Y})$ e coordenadas geográficas (UTM) com GPS MAP76, Garmim, USA. O potencial volumétrico de madeira das espécies amostradas foi avaliado a partir da frequência de indivíduos por classe de diâmetro pré-comercial (DAP $<50 \mathrm{~cm}$ ) e comercial $(\mathrm{DAP} \geq 50 \mathrm{~cm})$, mediante o cálculo pela equação 4 .

$$
V C=\pi \cdot \frac{D A P^{2}}{4} \cdot A C \cdot F C
$$

Em que: $V C=$ volume comercial $\mathrm{em} \mathrm{m}^{3} ; \pi=$ pi, equivalente a $3,14 \ldots ; D A P=$ diâmetro a $1,30 \mathrm{~m}$ do solo, em m; $A C=$ altura comercial, em $\mathrm{m} ; F C$ = fator de correção, equivalente a 0,7 .

Em virtude da baixa precisão métrica de aparelhos de GPS no interior da floresta e do alto custo de imagens de alta resolução espacial, foi desenvolvido o método de correção de coordenadas espaciais (MCCE), método de correção manual da localização das coordenadas UTM das árvores em concordância com a localização de campo ou cartesianas $(\mathrm{X}, \mathrm{Y})$, aliado ao cálculo do índice do vizinho natural (ANND) no reconhecimento e análise de padrões espaciais de espécies em florestas nativas da Amazônia. Uma alternativa a essa situação é o uso da tecnologia Light Detection and Ranging - Lidar (Koch, 2012; Wezyk, 2012), que apresenta relativa precisão dependendo do objetivo, no entanto, devido ao elevado custo ainda é inacessível para a maioria das instituições de pesquisa na Amazônia.

O método MCCE foi realizado em cinco passos (Figura 1): 1ํㅡ) No ArcGIS foi criada uma grade com células de $100 \mathrm{~m}$ x $100 \mathrm{~m}$, subdivididas em 100 subparcelas de $100 \mathrm{~m}^{2}(10 \mathrm{~m} \times 10 \mathrm{~m})$, a partir da criação de um polígono contendo os vértices da parcela tomados em campo com GPS; $2^{\circ}$ ) Essa grade foi alocada no centro da nuvem de pontos (árvores) amostrados na parcela com o GPS (cruzes), sendo orientada pelos vértices tomados em campo; $3^{\circ}$ ) Através da combinação de informações de localização $(\mathrm{X}, \mathrm{Y})$ das árvores e das coordenadas UTM (ArcGIS), foi possível corrigir manualmente cada coordenada UTM dentro da grade, gerando uma localização espacial coincidente com a localização obtida em campo (X,Y); $4^{\circ}$ ) Em planilha eletrônica, foram 
analisados os desvios (em m) de cada coordenada UTM; $5^{\circ}$ ) Foram obtidas as coordenadas espaciais (UTM) coincidentes com a localização de campo ou coordenada cartesiana $(\mathrm{X}, \mathrm{Y})$. Este processo faz-se necessário para alocar dentro das parcelas os indivíduos (DAP $\geq 10 \mathrm{~cm}$ ) que foram posicionados fora da grade amostral e/ou deslocados de suas subparcelas de origem, em virtude do erro de localização do GPS. A realocação manual destes pontos é um critério subjetivo, porém efetivo em relação ao posicionamento espacial das amostras e suas estimativas de parâmetros dendrométricos (diâmetro, altura, volume, etc.), estando em concordância com as coordenadas cartesianas, contribuindo com o manejo florestal de precisão.

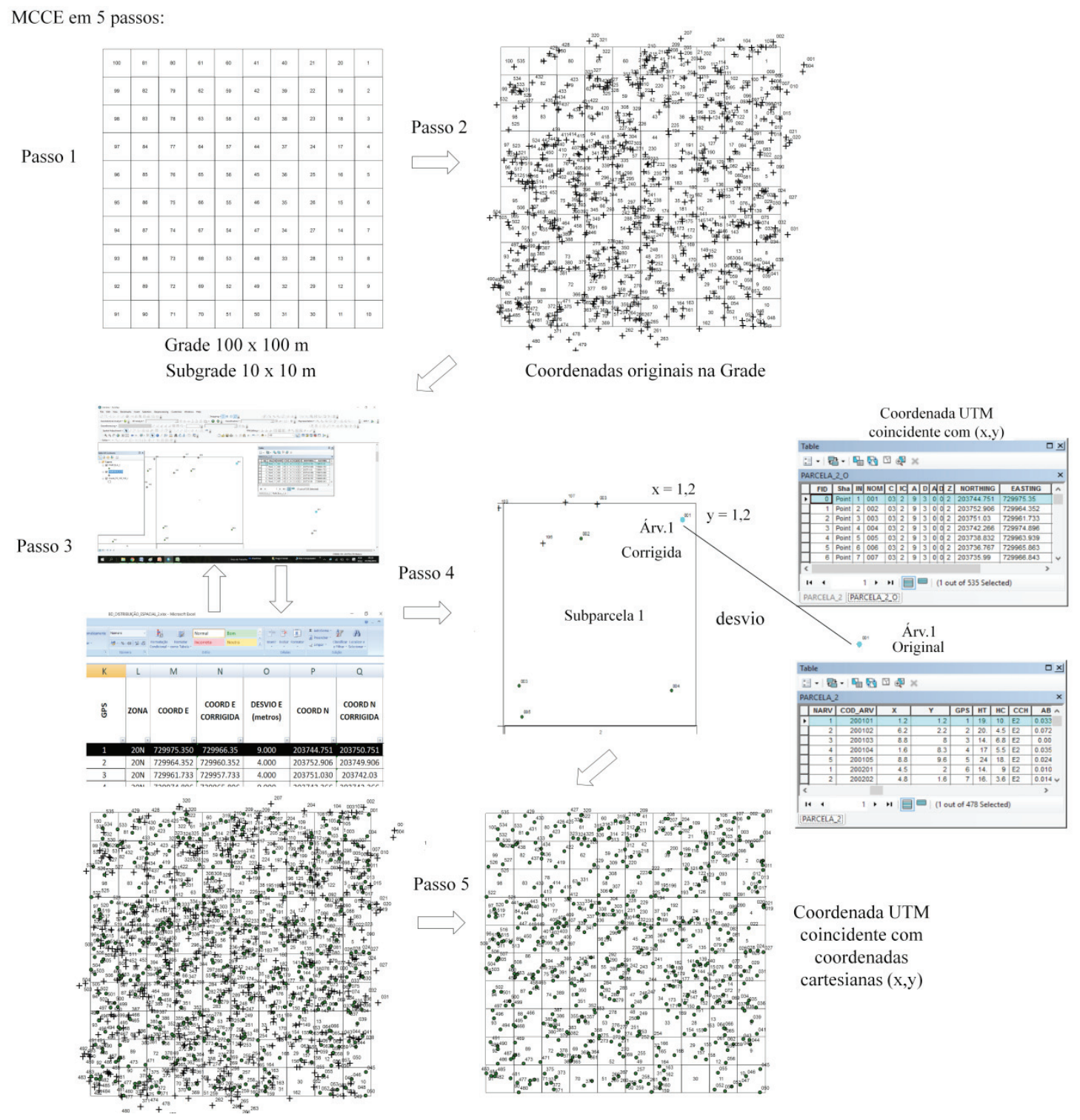

Figura 1. Método de coordenadas cartesianas e espaciais (MCCE) descrito em cinco passos. Em que: 1º) criação da grade de 1 ha subdividida em subparcelas de $\left.100 \mathrm{~m}^{2}(10 \times 10 \mathrm{~m}) ; 2^{\underline{o}}\right)$ alocação no centro da nuvem de pontos (árvores com DAP $\geq 10 \mathrm{~cm}$ ) amostradas por GPS; 3º) correção manual por planilha eletrônica e ArcGIS; $4^{\circ}$ ) análise dos desvios individuais; $5^{\circ}$ ) obtenção das coordenadas espaciais (UTM) coincidentes com a localização em campo $(\mathrm{X}, \mathrm{Y})$. 
A identificação botânica foi realizada em campo por especialistas do Instituto Nacional de Pesquisas da Amazônia. As árvores foram agrupadas em três estratos verticais de altura total (AT) (Condé \& Tonini, 2013): estrato inferior (EI, no qual $\mathrm{AT}<12,41 \mathrm{~m}$ ), estrato médio (EM, 12,41 $\mathrm{m} \leq \mathrm{AT}<26,46 \mathrm{~m}$ ) e estrato superior (ES, $\mathrm{AT} \geq 26,46 \mathrm{~m})$. Após a correção da localização espacial dos indivíduos arbóreos com DAP $\geq 10 \mathrm{~cm}$ por MCCE, foram realizadas simulações do ambiente florestal em formatos bi (2D) e tridimensional (3D), a partir da frequência de indivíduos por espécie por ha nos estratos verticais, utilizando os softwares ArcGIS 9.3 (ArcMap e ArcScene) e planilha eletrônica.

\section{Resultados e discussão}

Foram observados padrões espaciais predominantemente aleatórios para $E$. bracteosa e $M$. huberi e dispersos e raros foram predominantemente observados para C. cateniformis e D. excelsa (Tabela 1).

Tabela 1. Estimativas de padrões espaciais para quatro espécies madeireiras em 9 ha de floresta tropical densa em Caracaraí, RR.

\begin{tabular}{|c|c|c|c|c|c|c|c|c|}
\hline Parc. & Espécie & $\mathbf{N}$ & DO & DE & $\mathbf{z}$ & $\mathbf{p}$ & ANND & Distribuição \\
\hline \multirow{3}{*}{ P1 } & Cedrelinga cateniformis & 2 & 70,99 & 35,36 & 2,73 & $0,006^{* *}$ & 2,01 & Dispersa \\
\hline & Eschweilera bracteosa & 67 & 6,56 & 6,11 & 1,15 & $0,259^{\text {ns }}$ & 0,25 & Aleatória \\
\hline & Manilkara huberi & 10 & 16,30 & 15,81 & 0,19 & $0,852^{\mathrm{ns}}$ & 1,03 & Aleatória \\
\hline \multirow{4}{*}{$\mathbf{P 2}$} & Cedreling a cateniformis & 4 & 47,59 & 25,00 & 3,46 & $0,001 * *$ & 1,90 & Dispersa \\
\hline & Dinizia excelsa & 2 & 24,99 & 35,35 & 0,79 & $0,428^{\mathrm{ns}}$ & 0,71 & Aleatória \\
\hline & Eschweilera bracteosa & 45 & 8,11 & 7,45 & 1,13 & $0,259^{\text {ns }}$ & 1,09 & Aleatória \\
\hline & Manilkara huberi & 6 & 25,31 & 20,41 & 1,12 & $0,261^{\mathrm{ns}}$ & 1,24 & Aleatória \\
\hline \multirow{4}{*}{ P3 } & Cedrelinga cateniformis & 1 & - & - & - & - & - & Rara \\
\hline & Dinizia excelsa & 1 & - & - & - & - & - & Rara \\
\hline & Eschweilera bracteosa & 44 & 8,48 & 7,54 & 1,59 & $0,112^{\mathrm{ns}}$ & 1,13 & Aleatória \\
\hline & Manilkara huberi & 4 & 34,12 & 25,00 & 1,40 & $0,163^{\mathrm{ns}}$ & 1,36 & Aleatória \\
\hline \multirow{2}{*}{ P4 } & Eschweilera bracteosa & 43 & 7,62 & 7,62 & $-0,01$ & $0,993^{\text {ns }}$ & 1,00 & Aleatório \\
\hline & Manilkara huberi & 7 & 24,41 & 18,90 & 1,48 & $0,140^{\mathrm{ns}}$ & 1,29 & Aleatório \\
\hline \multirow{3}{*}{ P5 } & Dinizia excelsa & 1 & - & - & - & - & - & Rara \\
\hline & Eschweilera bracteosa & 60 & 6,81 & 6,45 & 0,81 & $0,416^{\mathrm{ns}}$ & 1,05 & Aleatória \\
\hline & Manilkara huberi & 1 & - & - & - & - & - & Rara \\
\hline \multirow{3}{*}{ P6 } & Dinizia excelsa & 1 & - & - & - & - & - & Rara \\
\hline & Eschweilera bracteosa & 68 & 6,26 & 6,06 & 0,51 & $0,609^{\text {ns }}$ & 1,03 & Aleatória \\
\hline & Manilkara huberi & 2 & 18,97 & 35,36 & 1,25 & $0,210^{\mathrm{ns}}$ & 0,54 & Aleatória \\
\hline \multirow{3}{*}{ P7 } & Cedrelinga cateniformis & 2 & 19,60 & 35,36 & $-1,21$ & $0,228^{\mathrm{ns}}$ & 0,55 & Aleatório \\
\hline & Eschweilera bracteosa & 67 & 7,30 & 6,11 & 3,07 & $0,002 * *$ & 1,20 & Dispersa \\
\hline & Manilkara huberi & 5 & 32,77 & 22,36 & 1,99 & $0,046^{*}$ & 1,47 & Dispersa \\
\hline \multirow{3}{*}{ P8 } & Dinizia excelsa & 1 & - & - & - & - & - & Rara \\
\hline & Eschweilera bracteosa & 84 & 5,30 & 5,46 & 0,51 & $0,612^{\mathrm{ns}}$ & 0,97 & Aleatório \\
\hline & Manilkara huberi & 12 & 18,21 & 14,43 & 1,73 & $0,083^{\text {ns }}$ & 1,26 & Aleatório \\
\hline \multirow{2}{*}{ P9 } & Eschweilera bracteosa & 50 & 8,03 & 7,07 & 1,84 & $0,065^{\text {ns }}$ & 1,14 & Aleatório \\
\hline & Manilkara huberi & 8 & 26,11 & 17,68 & 2,58 & $0,010^{* *}$ & 1,48 & Dispersa \\
\hline
\end{tabular}

Onde: $\mathrm{N}=\mathrm{n}^{\mathrm{o}}$ de indivíduos por ha (ind ha ${ }^{-1}$ ); $\overline{D O}$ = distância média observada entre um ponto ou amostra e seu vizinho mais próximo; $\overline{D E}$ = distância média esperada entre pontos ou amostras com padrão aleatório de seleção; $\mathrm{z}=$ desvio padrão; $\mathrm{p}$ = probabilidade associada à distribuição normal padrão com determinado nível de confiança $(90 \%, 95 \%$ e $99 \%) ;=$ proporção ou taxa média do índice do vizinho natural, em porcentagem;* $\mathrm{e}^{* *}=$ significativo a $95 \%$ e 99\%, respectivamente, sendo aceita a hipótese nula de aleatoridade; ns = não significativo, rejeita a hipótese nula de aleatoridade. 
Padrões espaciais específicos encontrados em florestas são guiados por dependência espacial de variáveis florestais (densidade, área basal, altura, volume e biomassa, etc.), ou seja, refletem a ocupação dos espaços e usos dos recursos disponíveis no ambiente pelas espécies, condicionadas pelos diferentes níveis de competição (Archanjo et al., 2012), pela forma e arquitetura das espécies, qualidade do sítio, luminosidade (Pritchard \& Comeau, 2004), distância entre árvores e aberturas na floresta (Sanquetta et al., 2007; Souza et al., 2012; Amaral et al., 2013). Estes padrões podem ser melhor analisados individualmente, por meio de ferramentas de SIG como ANND, análise de cluster, Kernel, etc. (Environmental Systems Research Institute, 2008; Amaral et al., 2013). A luminosidade é função da composição florística do estádio sucessional (Maass et al., 1995), podendo ter sua distribuição regulada pela composição florística e fitossociológica, como também pela própria topografia, que direciona a radiação solar, definindo habitats adequados a cada espécie vegetal na paisagem (Bian \& Walsh, 1993; Pillar, 2003). A dispersão de diásporos é de suma importância na propagação e estabelecimento de padrões das espécies vegetais, sendo a zoocórica mais efetiva em relação a anemocórica e a barocórica para espécies vegetais em florestas tropicais (van der Pijl, 1982; Giehl et al. 2007).

Hutchings (1986) afirma que o padrão espacial varia de acordo com o estrato analisado, tendendo a ser menos agregado em indivíduos adultos do que em plântulas, quando há alta taxa de mortalidade dependente de densidade (devido à ação de patógenos, herbívoros, etc.). Isso significa que, para uma escala espacial fixa, podemos ter diferentes padrões espaciais se estratificarmos a análise para cada espécie em diferentes estádios ontogenéticos. Foi observado para a maioria das espécies o padrão espacial aleatório, corroborando com outros estudos realizados em florestas tropicais (Rossi \& Higuchi, 1998; Oliveira \& Amaral, 2004). Entretanto, o comportamento de raridade de espécies foi observado em algumas parcelas, principalmente para $D$. excelsa e C. cateniformis, ou seja, espécies que apresentaram apenas 1 indivíduo por ha (Schilling \& Batista, 2008). Em se tratando de manejo florestal em áreas tropicais, a observância de alta raridade de espécies (Alves \& Miranda 2008; Condé \& Tonini 2013) implica em extrema cautela nas operações de planejamento e execução das atividades florestais.

Podemos observar na Figura 2 o resultado da simulação em ambiente bidimensional (2D) do cálculo do índice do vizinho natural aliado à distância euclidiana média
$(A N N D)$ para cada espécie comercial estudada em 1 ha de floresta nativa, referente à parcela 2 (P2), escolhida aleatoriamente entre as nove parcelas amostradas.
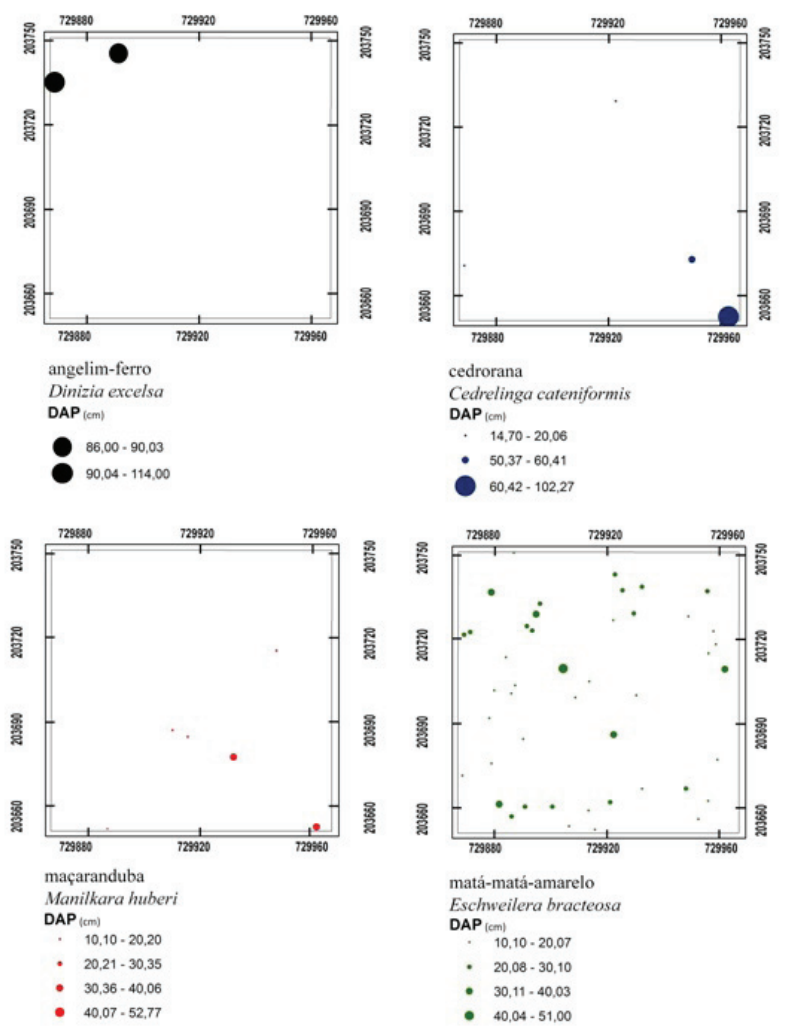

Figura 2. Padrões espaciais bidimensionais (2D) de quatro espécies madeireiras em 1 ha (parcela 2) de área de manejo florestal em Caracaraí, RR.

Oliveira \& Amaral (2004), utilizando o Índice de Morisita, observaram que E. bracteosa se apresentou de forma agrupada. Este gênero tem sido considerado hiperdominante em florestas amazônicas (ter Steege et al., 2013). Porém, uma espécie pode variar significativamente sua distribuição espacial em função da topografia, condições edafoclimáticas, gradientes ambientais e relações de dominância inter e intraespecíficas e interações bióticas (Brown et al., 2013; ter Steegeet al., 2013; Yang et al., 2013). No presente estudo, a distribuição observada para esta espécie não parece estar associada a variações abióticas, característica comum entre as espécies do gênero Eschweilera.

A espécie D. excelsa apresentou os maiores valores médios em diâmetro e altura comercial e total entre as quatro espécies comerciais analisadas. O georreferenciamento com GPS apontou em média erros de posicionamento da ordem de $5 \mathrm{~m}$, porém em alguns pontos o erro chegou a $45 \mathrm{~m}$ em relação à origem (Tabela 2). 
Tabela 2. Estimativas de variáveis florestais e desvios das coordenadas espaciais de quatro espécies comerciais madeireiras em 9 ha de floresta tropical densa em Caracaraí, RR.

\begin{tabular}{|c|c|c|c|c|c|c|c|c|c|c|c|c|}
\hline \multirow{2}{*}{ Espécie } & \multirow{2}{*}{$\mathbf{N}$} & \multirow{2}{*}{ DAP } & \multirow{2}{*}{ AC } & \multirow{2}{*}{ AT } & \multirow{2}{*}{ B } & \multirow{2}{*}{$\mathrm{VC}$} & \multicolumn{3}{|c|}{ Desvio N } & \multicolumn{3}{|c|}{ Desvio E } \\
\hline & & & & & & & Mín & Méd & Máx & Mín & Méd & Máx \\
\hline C. cateniformis & 9 & 45,8 & 15,1 & 32,7 & 2,11 & 24,74 & 0 & 4 & 12 & 1 & 3 & 5 \\
\hline D. excelsa & 6 & 74,4 & 20,2 & 36,7 & 3,13 & 50,31 & 4 & 7 & 12 & 1 & 6 & 8 \\
\hline E. bracteosa & 528 & 21,5 & 10,4 & 19,9 & 24,81 & 239,56 & 0 & 4 & 25 & 0 & 5 & 45 \\
\hline M. huberi & 55 & 38,7 & 15,4 & 28,6 & 9,85 & 142,72 & 0 & 5 & 16 & 0 & 5 & 14 \\
\hline Média & 150 & 45,1 & 15,3 & 29,5 & 9,97 & 114,33 & 1 & 5 & 16 & 1 & 5 & 18 \\
\hline Total & 598 & - & - & - & 39,89 & 457,33 & - & - & - & - & - & - \\
\hline
\end{tabular}

Em que: $\mathrm{N}=$ número de indivíduos amostrados; $\mathrm{DAP}=$ diâmetro a $1,30 \mathrm{~m}$ do solo $(\mathrm{cm}) ; \mathrm{AC}=$ altura comercial média $(\mathrm{m}) ; \mathrm{AT}=$ altura total média $(\mathrm{m}) ; \mathrm{B}=$ área basal $\left(\mathrm{m}^{2}\right)$; $\mathrm{VC}=$ volume comercial $\left(\mathrm{m}^{3}\right)$; Desvio $\mathrm{N}$ - mínimo (Mín), médio (Méd) e máximo (Máx) da coordenada Norte (N) em relação ao ponto original (m); Desvio E - mínimo (Mín), médio (Méd) e máximo (Máx) da coordenada Leste (E) em relação ao ponto original (m).

Em termos de densidade e volumetria comercial (DAP $\geq 50 \mathrm{~cm})$, as espécies $M$. huberi $\left(2,2\right.$ ind. ha ${ }^{-1}$ e $\left.14,3 \mathrm{~m}^{3} \mathrm{ha}^{-1}\right)$ e E. bracteosa $\left(2,1\right.$ ind. ha ${ }^{-1}$ e $7,6 \mathrm{~m}^{3}$ $\mathrm{ha}^{-1}$ ) demonstraram maior potencial para a extração madeireira (Figura 3), considerando uma estrutura diamétrica equilibrada (Souza et al., 2012; Condé \& Tonini, 2013). As espécies $C$. cateniformis e D. excelsa devem ser analisadas com cautela, tendo em vista a baixa densidade de indivíduos por hectare, porém com volumetria apta para extração comercial $\left(0,4\right.$ ind. ha ${ }^{-1} \mathrm{e}$ 2,5 $\mathrm{m}^{3} \mathrm{ha}^{-1}$; 0,4 ind. ha ${ }^{-1} ; 5,3 \mathrm{~m}^{3} \mathrm{ha}^{-1}$, respectivamente). Em se tratando de manejo florestal na Amazônia, os cuidados devem ser redobrados, em virtude da importância de extração vegetal amparada nos critérios de sustentabilidade e legalidade (Brasil, 2006), visando a perpetuação deste grande potencial biológico.
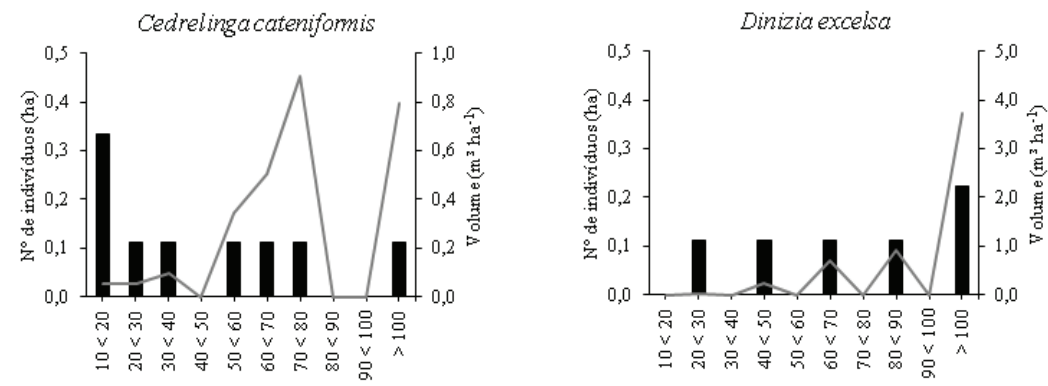

Classe de diâmetro $(\mathrm{cm})$

Classe de diâm etro (cm)
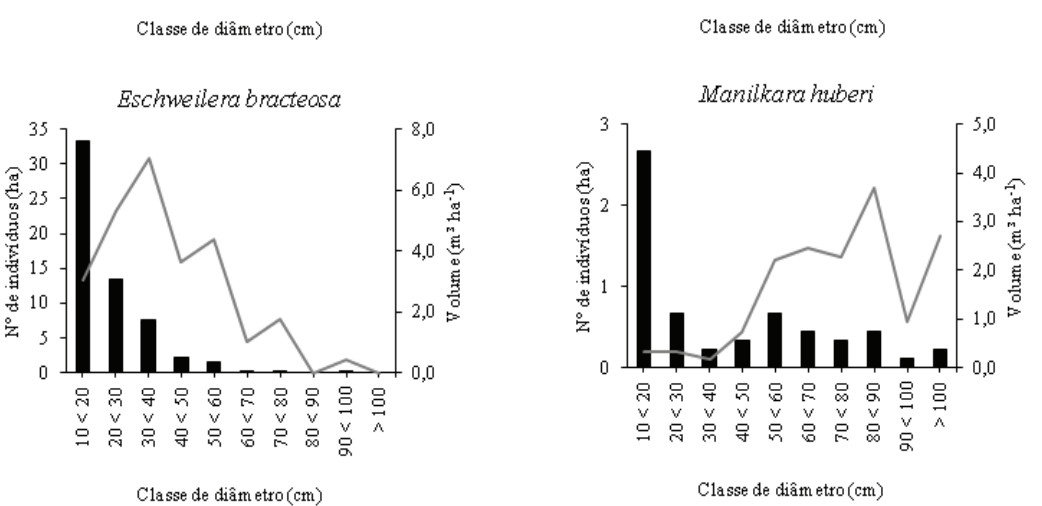

Classe de diâm etro $(\mathrm{cm})$

Figura 3. Frequência de indivíduos e volume de madeira por espécie e por classe de diâmetro por hectare em Caracaraí, RR. Em que: barras pretas = frequência de indivíduos por hectare; linha cinza $=$ volume por hectare. 
Neste estudo, a maioria dos indivíduos amostrados apresentou diâmetro inferior ao DMC (DAP $\geq 50 \mathrm{~cm})$, conforme a Instrução Normativa $n^{\circ} 5$ (Brasil, 2006). Isto demonstra a importância do método desenvolvido (MCCE) em análise conjunta com a distribuição diamétrica (Figura 3 ) e estratificação vertical na detecção dos verdadeiros padrões espaciais das espécies presentes em florestas tropicais. Indivíduos destas classes de diâmetro, por ocorrerem em grande número na floresta, contribuem majoritariamente para os erros de alocação e consequentemente erros na determinação dos padrões de distribuição espacial. A estratificação vertical da floresta permitiu a melhor visualização do grau de ocupação e dominância das espécies nos respectivos estratos verticais da floresta, sendo bastante útil na etapa de modelagem tridimensional (Figura 4). No estrato inferior dos 9 ha estudados, foi observado E. bracteosa (49) e M. huberi (2). O estrato médio foi composto por C. cateniformis (3), D. excelsa (2), E. bracteosa (392) e M. huberi (27). O estrato superior era composto por C. cateniformis (6), D. excelsa (4), E. bracteosa (87) e $M$. huberi (26). Foi observada alta densidade de indivíduos de $E$. bracteosa em todos os estratos verticais, resultado similar ao encontrado por Oliveira et al. (2008) em floresta densa de terra firme no Amazonas. $\mathrm{O}$ alto grau de ocupação desta espécie em ambos os estratos verticais da floresta facilita sua propagação, germinação, desenvolvimento e reprodução.

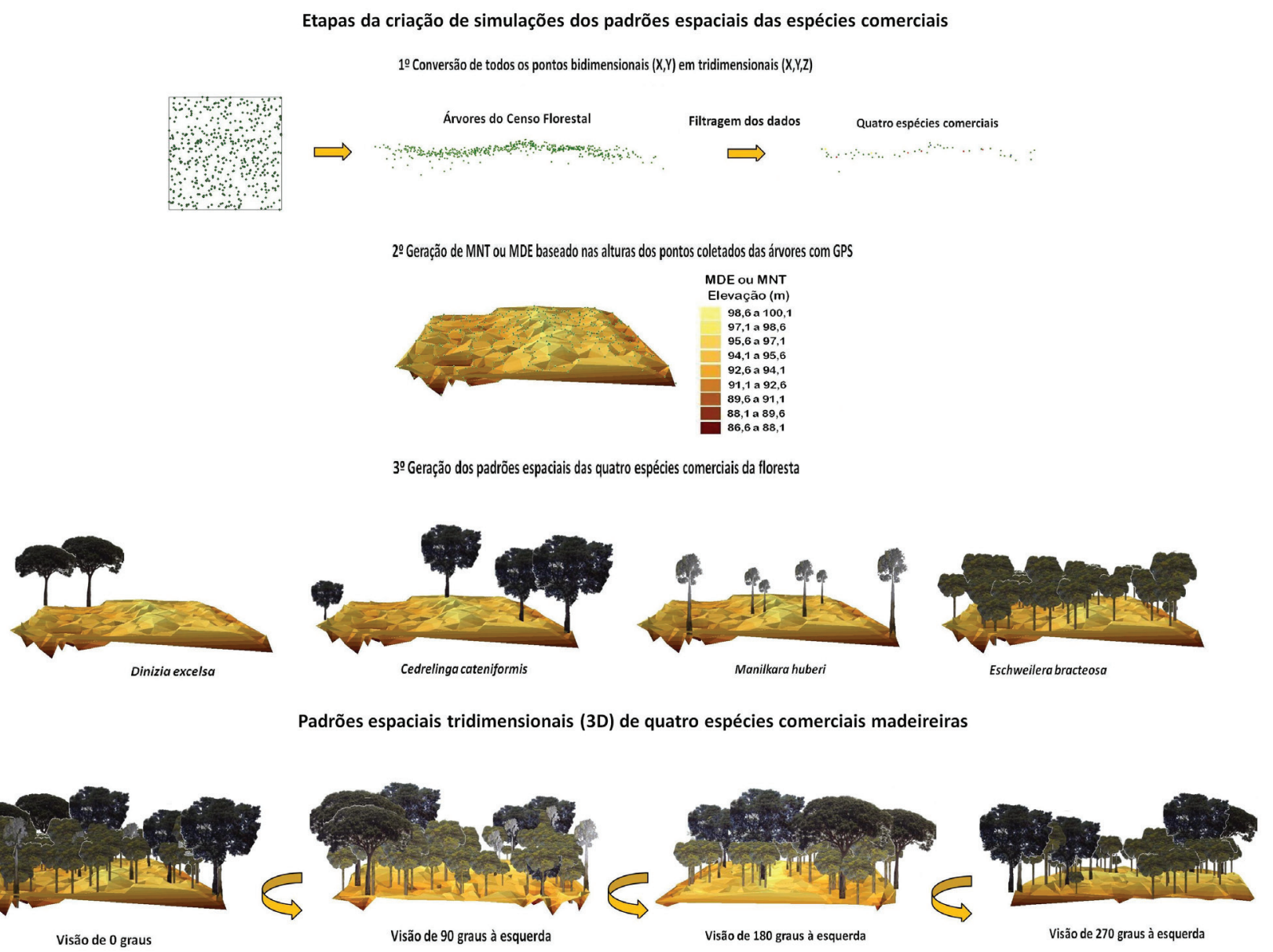

Figura 4. Padrões espaciais tridimensionais (3D) de quatro espécies comerciais madeireiras em 1 ha de floresta tropical densa em Caracaraí, RR. 
O método MCCE aliado ao ANND demonstrou ser eficiente para o reconhecimento e análise de padrões espaciais de espécies nativas da floresta tropical, podendo ser considerado uma alternativa ao manejo florestal de precisão na obtenção da localização precisa de árvores e suas estimativas florestais com diâmetros pré comerciais $(\mathrm{DAP}<50 \mathrm{~cm}$ ), que representam a grande maioria das árvores em florestas naturais (Souza et al., 2003, 2006; Condé \& Tonini, 2013). Desta forma, possui vantagem em relação à abordagem de métodos clássicos (Rossi \& Higuchi, 1998; Nascimento et. al., 2001; Queiroz et al., 2007), por possibilitar simulações e análises de padrões espaciais de espécies florestais em ambientes 2D e 3D. O padrão mais distinto foi apresentado por $D$. excelsa, formando apenas um agrupamento na área (Figura 5), com certa direcionalidade em função das regiões mais baixas (isotrópico). A maioria dos agrupamentos não apresentou direcionalidade, também chamada de anisotropia, com relação à disposição das curvas de nível. Brown et al. (2013) afirmam que a heterogeneidade de padrões espaciais das espécies em florestas tropicais pode estar intimamente ligada às variações ambientais, principalmente à topografia e suas relações interespecíficas na paisagem, sendo o uso da estatística espacial em ambientes de realidade virtual uma base promissora para a compreensão dos processos ecológicos sobre os nichos de espécies em ambientes heterogêneos.

As espécies E. bracteosa e M. huberi, aparentemente não têm suas distribuições espaciais influenciadas por fatores abióticos, como solo e topografia (Figura 4). Variações na topografia são frequentemente relacionadas a padrões de abundância de espécies arbóreas, em escala local, de diversas formações vegetais, ligadas à fertilidade, profundidade dos solos e lençol freático, drenagem e quantidade de serapilheira (Budkeet al., 2007). Entretanto, em áreas onde aparentemente as variações ambientais não limitam o desenvolvimento de uma determinada espécie, fatores biológicos como o crescimento vegetativo, composição dos estratos verticais e dispersão de diásporos (van der Pijl, 1982; Giehl et al., 2007), as interações intra e interespecíficas são normalmente citadas como responsáveis por agrupamentos em determinadas espécies (Perry \& Dixon 2002). A síndrome de dispersão parece determinar o padrão de distribuição de muitas espécies florestais. A dispersão zoocórica tem se mostrado como a estratégia mais eficaz, quando comparada às demais (van der Pijl,
1982; Giehl et al., 2007), associada a ninhos ou ao longo de rotas de animais frugívoros (Schuppet al., 2002).

Conhecer o padrão espacial pode ser o primeiro passo para investigar as variáveis que determinaram a configuração espacial e contribuíram para a distribuição natural das espécies (Dalmaso et al., 2012). Tecnologias avançadas de sensoriamento remoto permitem a acumulação de uma base de dados em três dimensões (3D), o que possibilita a modelagem ampla e realística de uma gama de variedades de eco-serviços florestais (Koch, 2012; Wezyk, 2012). A modelagem de parâmetros florestais em terceira dimensão quase não foi realizada até o momento, necessitando de aperfeiçoamento (Koch, 2012), principalmente em áreas de floresta tropical. Análises pontuais são de extrema importância, no entanto, a análise de séries temporais permitirão analisar mudanças e melhorar os modelos de vegetação, gerando informações fundamentais para compreensão e quantificação da produção de madeira, biomassa e carbono da floresta.

\section{Conclusões}

Em 9 ha de floresta sob manejo florestal em Caracaraí, RR, observou-se padrão espacial aleatório para $E$. bracteosa e $M$. huberi, ambas com potencial para extração comercial em virtude do equilíbrio na densidade e volumetria. Padrões espaciais dispersos e raros foram predominantemente observados em $C$. cateniformis e $D$. excelsa, exigindo maior cautela para o manejo dessas espécies.

MCCE demonstrou ser um método eficiente quando aliado ao ANND para o reconhecimento e análise de padrões espaciais de espécies nativas da floresta tropical, possibilitando a mitigação de erros de georreferenciamento de árvores pré comerciais (DAP $<50 \mathrm{~cm}$ ) e comerciais (DAP $\geq 50 \mathrm{~cm}$ ). Desta forma, possui vantagem em relação à abordagem de métodos clássicos, por possibilitar simulações e análises de padrões espaciais das espécies florestais em ambientes 2D e 3D da floresta, relacionando variáveis ambientais e contribuindo para o manejo florestal de precisão.

\section{Agradecimentos}

Agradecemos o apoio financeiro da Coordenação de Aperfeiçoamento Pessoal de Ensino Superior - Capes, Empresa Brasileira de Pesquisa Agropecuária - Embrapa (Roraima/Acre), com ênfase à colaboração do técnico 
Taiguara Pereira dos Santos, ao Programa de Mestrado em Recursos Naturais da Universidade Federal de Roraima - PRONAT e a Empresa Madeireira Vale Verde Ltda. em Caracaraí, RR.

\section{Referências}

Alves, J. C. Z. O. \& Miranda, I. S. Análise da estrutura de comunidades arbóreas de uma floresta amazônica de Terra Firme aplicada ao manejo florestal. Acta Amazonica, n. 38, n. 4, p. $657-$ 666, 2008. DOI: 10.1590/S0044-59672008000400008.

Amaral, L. P. et al. Influência da floresta alterada na distribuição espacial de três espécies da Floresta Ombrófila Mista avaliada pela geoestatística. Revista Árvore, v. 37, n. 3, p. 491-501, 2013. DOI: 10.1590/S0100-67622013000300012.

Archanjo, K. M. P. A. et al. Estrutura do componente arbóreo da Reserva Particular do Patrimônio Natural Cafundó, Cachoeiro de Itapemerim, Espírito Santo, Brasil. Floresta, v. 42, n. 1, p. 145$160,2012$.

Bian, L. \& Walsh, S. J. Scale dependencies of vegetation and topography in amountainous environment of Montana. Professional Geographer, v. 45, n. 1, p. 1-11, 1993. DOI: 10.1111/j.00330124.1993.00001.x.

Brasil. Instrução Normativa $\mathrm{n}^{\circ}$ 5, de 11 de dezembro de 2006. Dispõe sobre procedimentos técnicos para elaboração, apresentação, execução e avaliação técnica de Planos de Manejo Florestal Sustentável - PMFS's nas florestas primitivas e suas formas de sucessão na Amazônia Legal, e dá outras providências. Diário Oficial [da] República Federativa do Brasil, v. 143, n. 238, p. 155-159, 2006.

Brown, C. et al. Multispecies coexistence of trees in tropical forests: spatial signals of topographic niche differentiation increase whith environmental heterogeneity. Proceedings of Royal Society B, v. 280, n. 1764, p. 1-8, 2013. DOI: 10.1098/rspb.2013.0502.

Budke, J. C. et al. Relationships between tree component structure, topography and soils of a riverside forest, Rio Botucaraí, Southern Brazil. PlantEcology, v. 189, n. 2, p. 187-200, 2007. DOI: 10.1007/ s11258-006-9174-8.

Condé, T. M. \& Tonini, H. Fitossociologia de uma Floresta Ombrófila Densa na Amazônia Setentrional, Roraima, Brasil. Acta Amazonica, v. 43, n. 3, p. 247-260, 2013. DOI:10.1590/S004459672013000300002 .

Dalmaso, C. A. et al. Análise dos padrões espaciais de Ocotea odorifera (Vell.) Rohwer na floresta nacional de Irati, PR em ambiente SIG. Ambiência, v. 8, número especial, p. 559-570, 2012.

Environmental Systems Research Institute. ArcGIS 9.3 help: 3D analyst exercises index. [2008]. Avaliable from: $<\mathrm{http}: / /$ webhelp. esri.com/arcgisdesktop/9.3/index.cfm?TopicName=3D_Analyst Tutorial>. Access on: 15 Oct. 2013.

Fearnside, P. M. Global warming and tropical land-use change: greenhouse gas emissions from biomass burning, decomposition and soils in forest conversion, shifting cultivation and secondary vegetation. Climatic Change, v. 46, n. 1, p. 115-158, 2000. DOI: 10.1023/A:1005569915357
Figueiredo, E. O. et al. Manejo de precisão em florestas tropicais: modelo digital de exploração florestal. Rio Branco, AC: Embrapa Acre, 2007. 183 p.

Giehl, E. L. H. et al. Espectro e distribuição vertical das estratégias de dispersão de diásporos do componente arbóreo em uma floresta estacional no sul do Brasil. Acta Botanica Brasilica, v. 21, n. 1, p. 137-145, 2007. DOI: 10.1590/S0102-33062007000100013.

Gimenez, B. O. et al. Equações volumétricas para espécies comerciais madeireiras do sul do estado de Roraima. Scientia Forestalis, v. 43, n. 106, p. 291-301, 2015.

Hutchings, M. J. The structure of plant population. In: Crawley, M. J. (Ed.). Plant Ecology. Blackwell Scientific Publ., Oxford, 1986. p. $97-136$.

Jankauskis, J. Avaliação de técnicas de manejo florestal. Belém, PA: SUDAM, 1990. 143 p.

Krebs, C. Ecological methodology. 2. ed. Benjamin: Cummings, 1999. $620 \mathrm{p}$.

Koch, B. Modelling forest eco-services with multi-sensoral data. Ambiência, v. 8, número especial, p. 571-578, 2012.

Maass, J. M. et al. Seasonal changes of leaf area index (LAI) in a tropical deciduous forest in west Mexico. Forest Ecology and Management, v. 74, n. 1-3, p. 171-180, 1995. DOI: 10.1016/03781127(94)03485-F.

Nascimento, A. R. T. et al. Estrutura e padrões de distribuição espacial de espécies arbóreas em uma amostra de Floresta Ombrófila Mista em Nova Prata. Ciência Florestal, v. 11, p. 105-119, 2001.

Nogueira, E. M. et al. Estimates of forest biomass in the Brazilian Amazon: New allometric equations and adjustments to biomass from wood-volume inventories. Forest Ecology and Management, v. 256, n. 11, p. 1853-1867, 2008. DOI: 10.1016/j.foreco.2008.07.022.

Oliveira, A. N. \& Amaral, I. L. Florística e fitossociologia de uma floresta de vertente na Amazônia Central, Amazonas, Brasil. Acta Amazonica, v. 34, n. 1, p. 21-34, 2004. DOI: 10.1590/S004459672004000100004.

Oliveira, A. N. et al. Composição e diversidade florístico-estrutural de um hectare de floresta densa de terra firme na Amazônia Central, Amazonas, Brasil. Acta Amazonica, v. 38, n. 4, p. 627-642, 2008. DOI: $10.1590 /$ S0044-59672008000400005.

Perry, J. N. \& Dixon, P. M. A new method to measure spatial association forecological count data. Ecoscience, v. 9, n. 2, p. 133$141,2002$.

Pillar, V. D. Dinâmica de expansão florestal em mosaicos de floresta e camposno sul do Brasil. In: Cabido, M. (Ed.). Ecossistemas brasileiros: manejo econservação. Fortaleza: Expressão, 2003. p. 209-216.

Pritchard, J. M. \& Comeau, P. G. Effects of opening size and stand characteristics on light transmittance and temperature under young trembling aspen stands. Forest Ecology and Management, v. 200, n. 1-3, p. 119-128, 2004. DOI:10.1016/j.foreco.2004.06.002.

Queiroz, J. A. L. et al. Estrutura e dinâmica de floresta de várzea no estuário amazônico no estado do Amapá. Floresta, v. 37, p. 339-352, 2007. 
Ribeiro, J. E. L. S. et al. Flora da Reserva Ducke. Guia de identificação das plantas vasculares de uma floresta de terra firme na Amazônia Central. Manaus: INPA-DFID, 1999. 800 p.

Rossi, L. M. B. \& Higuchi, N. Comparação entre métodos de análise de padrão espacial de oito espécies arbóreas de uma floresta tropical úmida. In: Gascon, C. \& Moutinho, P. (Ed.). Floresta Amazônica: dinâmica, regeneração e manejo. Manaus: Instituto Nacional de Pesquisas da Amazônia, 1998. p. 41-60.

Sanquetta, C. R. et al. Dinâmica de um remanescente de Floresta Ombrófila Mista no sul do Paraná sob influência de taquaras. Ambiência, v. 3, n. 1, p. 65-78, 2007.

Schilling, A. C. \& Batista, J. L. F. Curva de acumulação de espécies e suficiência amostral em florestas tropicais. Revista Brasileira de Botânica, v. 31, n. 1, p. 179-187, 2008.

Schupp, E. W. et al. Dissemination limitation and theorigin and maintenance of species-rich tropical forests. In: Levey, D. J. et al. (Ed.). Seed dispersal and frugivory: ecology, evolution and conservation. New York: CAB International, 2002. p. 19-33

Serviço Florestal Brasileiro. Inventário florestal nacional - IFN. Brasília, DF, 2016. Disponível em: <http://www. http://ifn.florestal. gov.br/> Acesso em: 07 de março de 2016.

Silva, J.N.M. et al. Diretrizes para a instalação e medição de parcelas permanentes em florestas naturais da Amazônia Brasileira. Belém, PA: Embrapa Amazônia Oriental, 2005. 36 p.

Souza, D. R. et al. Emprego de análise multivariada para estratificação vertical de florestas ineqüiâneas. Revista Árvore, v. 27, n. 1, p. 5963, 2003. DOI: 10.1590/S0100-67622003000100008.
Souza, D. R. et al. Análise estrutural em floresta Ombrófila Densa de terra firme não explorada, Amazônia Oriental. Revista Árvore, v. 30, n. 1, p. 75-87, 2006. DOI: 10.1590/S0100-67622006000100010.

Souza, P. B. et al. Estrutura diamétrica dos estratos e grupos ecológicos de uma área de Floresta Estacional Semidecidual, em Dionísio, MG. Revista Árvore, v. 36, n. 1, p. 151-160,2012. DOI: 10.1590/S0100-67622012000100016.

ter Steege, H. et al. Continental-scale patterns of canopy tree composition and function across Amazonia. Nature, v. 448, p. 444447, 2006. DOI: 10.1038/nature05134.

ter Steege, H. et al. Hyperdominance in the Amazonian tree flora. Science, v. 342, p. 325-334, 2013. DOI:10.1126/science.1243092.

Turner, W. R. \& Tjørve, E. Scale-dependence in species-area relationships. Ecography, v. 28, p. 721-730, 2005.

van der Pijl, L. Principles of dispersal in higher plants. Berlin: Springer-Verlag, 1982. 214 p.

Wezyk, P. The integration of the terrestrial and airborne laser scanning technologies in the semi-automated process of retrieving selected trees and forest stand parameters. Ambiência,v. 8, número especial, p. 533-548, 2012.

Yang, J. et al. A Phylogenetic Perspective on the Individual SpeciesArea Relationship in Temperate and Tropical Tree Communities. PloS ONE, v. 8, e63192, 2013. DOI: 10.1371/journal.pone.0063192. 
